\title{
La crítica de Axel Honneth a la dicotomía habermasiana entre sistema y mundo de la vida
}

\author{
Axel Honneth's critique of the Habermasian dichotomy \\ between system and lifeworld
}

ANA CAROLINA FASCIOLI ALVAREZ

Universidad de la República (URUguay)

Artículo recibido: 15 marzo 2016

Solicitud de revisión: 16 marzo 2016

Artículo aceptado: 13 julio 2016

\begin{abstract}
Resumen
El presente artículo analiza la crítica de Axel Honneth a la distinción habermasiana entre sistema y mundo de la vida como una ficción teórica que acarrea un déficit explicativo de la dinámica social y una rebaja del propio enfoque comunicativo del autor. Se vincula esta crítica con la que han realizado otros teóricos y se valora su pertinencia a la luz de la réplica que el propio Habermas brindó. A partir de allí, se intenta mostrar cómo este distanciamiento de Honneth habilita una nueva forma -aunque no explorada por el autor- de conceptualizar el carácter y rol de la sociedad civil.
\end{abstract}

Palabras clave: Honneth, Habermas, sistema, mundo de la vida, sociedad civil, Teoría Crítica

\begin{abstract}
This article provides an analysis of Honneth's objection to Habermas's distinction between System and Lebenswelt. Honneth postulates it's a theorical fiction that leads to misunderstand social dynamics and weakens his communicative perspective. Honneth's objection is linked to other critical reviews of the distinction, and I conclude its relevance, also considering Habermas's reply. From there, I try to show how Honneth objection opens a new way to think about civil society.
\end{abstract}

Key words: Honneth, Habermas, System, Lebenswelt, civil society, Critical Theory. 


\section{INTRODUCCIÓN}

Como es sabido, la teoría de la acción comunicativa de Habermas tuvo el cometido de recuperar el proyecto de una teoría crítica de la sociedad, desde un cambio de paradigma de la idea misma de razón. Contra una visión reduccionista de la racionalidad, Habermas introdujo la noción de una racionalidad comunicativa como el conjunto de las pretensiones de validez presentes en todo agente que actúa lingüísticamente con vistas a entenderse con otros.A diferencia de la racionalidad meramente instrumental orientada al éxito, la comunicativa abandona la esfera individual y sitúa el foco de la acción en la cooperación entre los sujetos (Habermas, 1987).

Estas dos formas de racionalidad se dan integradas en una doble dimensión de acción social que Habermas describe como «sistema» (System) y «mundo de la vida» (Lebenswelt). Esta distinción representa el corazón o núcleo del concepto de sociedad desarrollado por Habermas. Una sociedad, como conjunto activo de individuos que cooperativamente pretenden reproducir, mantener y mejorar sus condiciones de vida, se constituye e integra en dos dimensiones: por un lado, como ámbito de integración intersubjetiva o «integración social» y, por otro lado, como acción coordinada dirigida a fines o «integración sistémica». Esta segunda dimensión es el conjunto funcional observable mediante el que los miembros de una sociedad desarrollan su acción, guiados por criterios racionales adecuados al control de sus circunstancias vitales. Junto al sistema, e incorporándolo en un contexto más amplio, se encuentra el ámbito de la integración intersubjetiva: el conjunto de estructuras comunicativas, lingüísticamente articuladas, mediante las cuales los hombres establecen el acuerdo básico que rige su cooperación. Este presupuesto básico y horizonte incuestionado de la acción comunicativa es lo que llama mundo de la vida, recurriendo a un término fenomenológico acuñado por Husserl. Éste es el trasfondo -de patrones de socialización, valores, normas, etc.- implícitamente reconocido como válido que define nuestras posibilidades de actuar comunicativamente, estableciendo los presupuestos de toda racionalidad -tanto la instrumental como la comunicativa (Habermas, 1987; 2003).

Han sido numerosos los teóricos que han presentado críticas a tal famosa distinción entre sistema y Lebenswelt. En este artículo, se ofrece un análisis de la particular crítica de Axel Honneth, representante de la tercera generación de la Teoría Crítica. La teoría de Honneth se presenta en parte como continuidad y en parte como renovación del viraje comunicativo que Habermas introdujo en esta tradición de pensamiento. Su enfoque 
ha cobrado un creciente protagonismo en la reflexión académica, y su teoría del reconocimiento es referencia fundamental para la filosofía moral, política y social contemporánea.

Honneth reconoce que su trabajo continuó la ruta abierta por Habermas para sacar a la teoría crítica del estancamiento en que se encontraba y hallar una solución al problema de fundamentación de los estándares de la crítica social. La transformación comunicativa de la teoría realizada por Habermas puso a la vista una dimensión de la acción social -en la forma de expectativas normativas de interacción- en la cual se podía asentar la crítica (Honneth, 1995). Sin embargo, también tempranamente, Honneth entendió que la perspectiva de Habermas presentaba importantes insuficiencias y esto marcó un distanciamiento.

Aunque son diversas las críticas que éste ha presentado al enfoque habermasiano (Deranty, 2009), analizar cada una de ellas excedería las posibilidades de este artículo. Lo que aquí me propongo es presentar y valorar una crítica fundamental: la crítica de Honneth a la tajante distinción habermasiana entre sistema y mundo de la vida; una «ficción teórica» que, según el autor, acarrea un déficit explicativo de la dinámica social y de lo que constituyen rasgos patológicos de la misma. Ello trae consigo la concepción de dos sistemas autónomos de acción sin conexión entre sí: un dominio de interacción estratégico libre de normas y un mundo de la vida libre de poder. La patología de nuestra sociedad es vista como la penetración de formas sistémicas en la región previamente intacta de la práctica comunicativa cotidiana. Como consecuencia más relevante, queda invisibilizada la conflictiva interna del mundo de la vida y el sustento que el propio consenso normativo de los agentes otorga al funcionamiento sistémico. Junto a este déficit en el diagnóstico social de Habermas, en el artículo se señala de qué forma cuestionar tal dicotomía abre la puerta a reconceptualizar el papel y carácter de la sociedad civil en el marco de una teoría crítica de la sociedad. El artículo tiene también el valor de exponer los rasgos del pensamiento de Honneth en una etapa previa a la creación de su conocida obra La lucha por el reconocimiento.

\section{LA CRÍTICA DE HONNETH A HABERMAS EN CRÍTICA DEL PODER}

La referida crítica fue formulada muy tempranamente por Honneth, mientras elaboraba su tesis doctoral, y debemos retrotraernos a su primer 
libro Crítica del poder de 1985 para reconstruirla. El título de la obra se justifica porque allí Honneth ofrecía una comparación entre Adorno, Foucault y Habermas acerca de cómo interpretar las relaciones contemporáneas de dominación, y qué concepciones de la acción están en la base de la integración social y del ejercicio del poder. El libro tenía la novedad de ofrecer una provocativa interpretación de la historia de la Teoría Crítica como la historia de un aprendizaje, así como un análisis comparativo de los dos «nuevos» enfoques más influyentes y en competencia que ofrecía la teoría social crítica como solución a las aporías de la primera generación. Estos dos enfoques eran -para Honneth- la teoría del poder de Foucault, basada en sus investigaciones históricas, y la teoría de la sociedad de Habermas, basada en una teoría de la acción comunicativa (Honneth, 2009). En esa época, Honneth se encontraba intentando esbozar qué consideración de lo social y qué tipo de crítica del poder debería ofrecer una teoría a la vez normativa y práctica, para superar las dificultades que presentaban, a su vez, estos dos enfoques. Así, la obra que se publicó en 1985 fue valorada como una contribución significativa en el debate sobre los fundamentos normativos, esto es, sobre cómo asegurar reflexivamente los propios estándares críticos de una teoría social. En el prólogo a La lucha por el reconocimiento Honneth sostiene que este momento de su trayectoria fue el que lo orientó hacia el concepto de «lucha social éticamente motivada», que luego profundiza con un estudio sistemático de la noción hegeliana de una «lucha por el reconocimiento» (Honneth, 1997).

En Crítica del poder, Honneth denunciaba, siguiendo a Habermas, que la Teoría Crítica de la primera generación no pudo captar los procesos de integración, orientación social y el conflicto cotidiano, porque la lógica de dominio se suponía reproduciéndose con independencia de los sujetos. Particularmente Adorno había fallado en un análisis de la sociedad porque quedó encerrado en un modelo totalitario de dominio sobre la naturaleza, no pudiendo captar «lo social» de modo específico (Honneth, 2009). Asimismo, Honneth mostraba cómo las teorías críticas de Foucault y Habermas sí lograron abrir sus teorías a la acción social y realizó entonces una comparación entre ellos. Aunque Foucault incluyó el dinamismo de la interacción social y mostró la importancia del conflicto en el ejercicio cotidiano del poder, terminó excluyendo a los sujetos agentes ya que se volcó a un análisis sistémico del poder, por el cual éste es visto como una propiedad autoexpansiva de los sistemas sociales, más que como producto de una lucha entre actores estratégicos. Aunque el origen del dominio social está en un proceso de lucha estratégica entre sujetos, no puede dar 
base para un fundamento reflexivo de sus pretensiones críticas, porque el dominio es concebido aparte de todo acuerdo normativo o intención moral, sujeto a una pura explicación sistémica (Honneth 2009). En su opinión, la teoría de la acción comunicativa de Habermas ofrecía un punto de vista más interesante para un análisis de lo social que Foucault, porque reconocía el rol del «entendimiento mutuo» tanto como el conflicto estratégico en formas legítimas e ilegítimas de poder. Honneth reconoce allí que Habermas, sin negar el poder sistémico, hizo emerger la posibilidad de un modelo de conflicto social basado en una teoría de la comunicación (Honneth 2009), y de esta forma, la herencia marxista de una doctrina sobre la lucha de clases podía ser conservada de una manera realista, algo que preocupaba en particular al joven Honneth.

Sin embargo, el autor dedicó el último capítulo de aquella primera obra a cuestionar que Habermas termina siendo poco consecuente con aquel modelo intersubjetivo que él mismo introdujo. En el desarrollo de su teoría social, Habermas convirtió las intuiciones iniciales sobre la comunicación, subyacentes en su teoría del conocimiento, en dos concepciones sobre el orden social que suponen, a su vez, dos ideas diferentes sobre el desarrollo de la especie y dos diagnósticos de las crisis sociales (Honneth, 2009).

Ocurre, dice Honneth, que es posible encontrar en las distintas obras de Habermas estos dos modelos de cambio social compitiendo entre sí. Por un lado, un modelo que surge de su crítica a la tesis tecnocrática basado en la idea de una doble reproducción de la sociedad con una esfera de acción instrumental y otra comunicativa -la distinción entre trabajo e interacción- y que deriva luego en postular un modelo de la sociedad bajo la fuerte dualidad sistema-mundo de la vida (Habermas, 1987; 2003). Por otro lado, un segundo modelo, deudor de su lectura de Marx y su crítica a él, y que es descrito como una «dialéctica moral de lucha de clase», según la cual los sujetos entran en conflicto acerca de la interpretación de normas y la distribución asimétrica del poder (Habermas, 1990). Según este modelo, el orden social se mantiene a través de relaciones comunicativas institucionalmente mediadas entre grupos que se encuentran moralmente integrados.

[...] la construcción conceptual de un sistema de acción organizado racionalmente conforme a fines, que es la perspectiva teórica central del primer planteamiento, es puesta en cuestión y sobrepasada por la concepción que subyace al segundo, a saber, la idea de una formación de consenso moral que acompaña al conjunto de la sociedad y organiza todos los dominios de acción (Honneth, 2009: 409-410). 
Habermas luego no continuará con el análisis de una dialéctica moral del conflicto de clase y en los años 70 elabora su teoría social a partir del enfoque formulado en su crítica a la tesis tecnocrática. Según Honneth, en Teoría de la acción comunicativa -obra con la que culmina este procesoHabermas declara su preferencia por el primer modelo (Honneth, 2009). El resultado es que «lo social» termina dividido en un dominio de interacción estratégica libre de normas y un mundo de la vida libre de poder.

\section{CONSECUENCIAS DE UNA FICCIÓN TEÓRICA}

La crítica de Honneth a Habermas se centra allí en este último asunto. La objeción consiste en que el diagnóstico habermasiano sobre las patologías de la sociedad contemporánea como colonización sistémica sobre el mundo de la vida se asienta en una construcción teórica problemática, que Habermas terminó erigiendo y eligiendo: un dualismo ficticio entre sistema y mundo de la vida. Esta construcción surge, según Honneth, de la explicación teórico-evolutiva de Habermas sobre la emergencia de las sociedades modernas, en términos de una separación entre dos esferas de acción contrapuestas, pero también de la distinción inicial que hizo -y en la que ésta se asienta- entre dos modelos de coordinación de la acción en el proceso de integración social, uno orientado al éxito y otro al entendimiento. ${ }^{1}$

Así, se genera la gran ficción de dos ámbitos separados o totalmente autónomos: una esfera de acción libre de normas -sistema- y una esfera de comunicación libre de poder -mundo de la vida. En palabras de Honneth:

\footnotetext{
Si las sociedades capitalistas se conciben de este modo como órdenes sociales en los que las categorías de sistema y mundo de la vida se contraponen como esferas de acción que han alcanzado respectiva autonomía, surgen dos ficciones complementarias; cabe suponer entonces la existencia (1) de organizaciones de acción vaciadas de sustancia normativa y de (2) esferas de comunicación vaciadas de poder. En estas dos ficciones, producidas por el concepto de sistema conectado a la teoría de la acción, se reproducen esos errores teóricos que nosotros ya habíamos reconocido anteriormente en la crítica habermasiana de la «tesis tecnocrática» (Honneth, 2009).
}

1 Esta interpretación de los motivos que llevan a Habermas a tal dualismo es tomado por Honneth de McCarthy (McCarthy, 1985:110-126; citado por Deranty, 2009: 113). 
El autor analiza en profundidad estas dos ficciones derivadas de la anterior y que claramente tienen una relación complementaria entre sí. Por un lado, la idea de sistemas de acción organizados desde una racionalidad instrumental supone que

[...] las formas de organización de la economía y la administración del Estado deben comprenderse aún sólo como materializaciones de reglas de acción racional conforme a fines $\mathrm{y},[\ldots]$ que los rendimientos de la acción dentro de las organizaciones pueden tener lugar independientemente de los procesos orientados a la formación de consenso normativo (Honneth, 2009: 437).

Honneth piensa, en cambio, que resulta más acertado concebir las estructuras organizativas de la administración como encarnaciones tanto de principios instrumentales como práctico-políticos. Los estándares políticoprácticos que en cualquier momento determinan las condiciones normativas, bajo las cuales las tareas organizacionales son instrumentalmente alcanzadas, pueden ser concebidos como el resultado de un continuo proceso de comunicación entre los actores involucrados. Las acciones de dirección y administración producidas en las organizaciones permanecen dependientes de prácticas de entendimiento social, en el sentido de que no pueden ser conectadas a una serie de logros funcionales sin la mediación de decisiones situadas de los sujetos involucrados. La organización está sometida a un proceso de formación de consenso normativo porque su dominio específico de operación y su particular forma de organización están continuamente siendo tratados desde una nueva perspectiva. Por lo tanto, ni la dirección ni la administración pueden ser hechas independientemente de acuerdos normativos de sus miembros al punto que supone la sociología sistémica de las organizaciones (Honneth, 2009). Por otro lado e inversamente, la imagen de esferas de acción integradas comunicativamente, sugiere la independencia del mundo de la vida de prácticas de dominio y procesos de poder. ${ }^{2}$ Parece como si éste se reprodujera independientemente de las prácticas de influencia estratégica -o sea, del ejercicio físico, psicológico o cognitivo del poder-; como si sólo acciones orientadas al entendimiento generaran las coordinaciones de planes de acción en las esferas de comunicación que Habermas atribuye a la familia y a la esfera pública.

2 Honneth ya había anticipado esta crítica a Habermas por perder conexión con una teoría del conflicto de clase al desconocer las asimetrías de los procesos comunicativos cotidianos (Honneth, 1995b). 
Honneth reconoce que Habermas habría intentado en algún momento distinguir entre «acuerdo» e «influencia» en la propia coordinación lingüística de la acción, abriendo la posibilidad de distinguir formas de ejercicio del poder en el nivel de la integración social (Habermas, 1985). Pero en Teoría de la acción comunicativa esta idea se desestima, porque la influencia externa de los sujetos es considerada fundamentalmente en el nivel de los medios no lingüísticos de comunicación y en relación a la integración sistémica. Entonces, la categoría de integración social se asocia a formas de coordinación de la acción orientadas sólo al entendimiento, mientras la integración sistémica sólo hace referencia a formas de coordinación externa de la acción, orientadas al éxito. En palabras del autor:

\begin{abstract}
«Poder», aquí, que sólo es concebido en el ámbito de la integración sistémica como un medio de coordinación de la acción social, por lo que todos los procesos presistémicos de constitución y reproducción de dominio tienen que desaparecer del horizonte; por otro lado, los rendimientos de integración social del mundo de la vida se observan de tal forma en esas esferas de acción social que sirven a la tarea de la reproducción simbólica de una sociedad que todos los procesos de formación de consenso moral internos a una organización tienen, asimismo, que desaparecer (Honneth, 2009: 441).
\end{abstract}

\title{
3. UN HABERMAS POCO CONSECUENTE
}

Honneth encuentra que, con la primera ficción, Habermas contradecía los logros de su teoría comunicativa de la acción, al no considerar la importancia de formas pre-estatales, situacionales de ejercicio del dominio cotidiano en la reproducción de la sociedad, algo que la teoría del poder de Foucault si consideraba. Con la segunda ficción, Habermas contradecía todo lo que puede aprenderse de la crítica teórica comunicativa a la sociología clásica de las organizaciones: la importancia de los procesos de interacción interna a una organización para el funcionamiento de la misma (Honneth, 2009). La crítica entonces concluye que con estas ficciones termina abandonando intuiciones valiosas que el mismo Habermas introdujo.

Resulta interesante la reconstrucción que Honneth realiza sobre cómo es que Habermas llega a establecer que lo social quede divorciado en dos ámbitos mutuamente excluyentes, en el curso de la construcción de su teoría social. Según Honneth, ya existía una tendencia reificante en la crítica de Habermas a la tesis tecnocrática -diferenciando acción instrumental y comunicativa asociadas a esferas enteras de la vida social- que reaparece en este dualismo sistema-mundo de la vida. Al ir reformulando su teoría de la ac- 
ción, trabajo e interacción ya no se oponen directamente una a la otra como dos tipos de acción simplemente, más adelante, las acciones sociales son distinguidas por el mecanismo a través del cual son socialmente coordinadas (Honneth, 2009). Así, surgen dos formas de coordinación de la acción social en dos esferas de reproducción social. Lo que hace Habermas es entonces, continuar, en un nivel conceptual más avanzado, el dualismo que introdujo inicialmente. El diagnóstico social que resulta de este renovado dualismo -la idea de colonización del mundo de la vida por parte del sistema- presenta de una forma más precisa su previo análisis sobre la independencia de la tecnología como amenaza a la práctica comunicativa. En el recorrido del desarrollo de su teoría social, Habermas termina haciendo un diagnóstico de época que, como el de Adorno o Foucault, se concentra en las consecuencias de los poderes que se vuelven autónomos.

Sin embargo, al coordinar de forma tan rígida las dos dimensiones de la reproducción, la
material y la simbólica, con los mecanismos analíticamente diferentes de la integración so-
cial y sistémica se introducen en principio las mismas ficciones engañosas que ya estaban
de algún modo presentes en la primera aproximación al problema; es decir, esta distinción
en realidad también incorpora una diferencia en el proceso de reproducción social para la
que no se puede hallar ninguna correspondencia objetiva [...] (Honneth, 2009: 430).

Esta crítica a la ficticia dicotomía lleva así a una crítica derivada acerca del déficit de Habermas en el diagnóstico de la patología social, al entenderla como colonización unidireccional del sistema sobre un mundo de la vida previamente intacto (Habermas, 2003). Honneth postula de cierto modo -aunque sin mayor desarrollo teórico de la idea- que existe una mayor porosidad e interdependencia entre estas esferas o dimensiones. ¿Cómo lo argumenta? En una entrevista brindada en 2005, Honneth afirma:

Esto se puede entender mejor hoy en día, que hace quizás treinta o cuarenta años, cuando Habermas escribió su libro, porque toda la discusión sobre el tema dejó muy claro que una organización efectiva de la economía vive de los recursos provenientes del mundo de vida y que los debe incluir en la administración. En el caso inverso, se aplica también a los ámbitos que Habermas adjudica al mundo de vida, esencialmente a la privacidad. Estos ámbitos fueron penetrados solamente en un estado tardío por procesos de colonización con elementos sistémicos, y estudios sociológicos o históricos pueden demostrar que estos mundos de vida vivieron desde muy temprano en el desarrollo de sus sistemas característicos de la absorción de recursos capitalistas (Honneth, 2005: 12).

Como ejemplo, Honneth brinda el de la familia y el amor, en la esfera de la privacidad, que se nutren de la utilización sistemática y creativa de artículos de consumo producidos en el mercado (Honneth, 2005). Asimismo, 
argumenta que la idea de que cierta tendencia a la colonización surgió en un momento tardío del desarrollo capitalista - con su carácter de movimiento unilateral- no es correcta, debido a que estas esferas se nutrieron mutuamente desde el comienzo, y no estaban separadas de la manera esbozada por Habermas. En este sentido, los «estudios sociológicos e históricos» a los que refiere y en que se apoya Honneth son los trabajos de $\mathrm{B}$. Moore (1978) sobre el proletariado inglés y el concepto de honor implícito en sus reivindicaciones. Para Honneth, esta sociología histórica de los movimientos sociales pone en evidencia el núcleo moral de las luchas sociales: los trabajadores no demandan simplemente aumentos salariales motivados por obtener más dinero y/o poder, sino que también lo demandan motivados por el afán de ser reconocidos como haciendo aportes valiosos a la reproducción social. La fuente motivacional de los movimientos de resistencia y protesta no son los intereses económicos, ni única ni principalmente, sino la experiencia de la violación del propio honor (Honneth, 2006). Por ende, también en el sistema anidan elementos normativos y «no podemos hablar de la colonización, sino de formas siempre nuevas de mezclarse e influirse mutuamente» (Honneth, 2005:12). Esta crítica se expande aquí, no sólo a Habermas, sino a toda la Teoría Crítica y su visión del capitalismo, que reducía las disposiciones de los sujetos al patrón único de la racionalidad instrumental.

En síntesis, esta crítica de Honneth consiste fundamentalmente en mostrar cómo, a partir de una conceptualización errónea de lo social, su diagnóstico de las patologías de la sociedad moderna resulta insuficiente. La contraposición dicotómica de sistema y mundo de la vida, y el énfasis en la colonización de uno por el otro, no permite detectar la conflictividad interna del mundo de la vida. La forma en que se conceptualiza a éste último da a entender que es una esfera de acción libre de prácticas de dominación y procesos de poder, y que se reproduce independientemente de las prácticas de influencia estratégica. Este dualismo lo ha llevado a ignorar que el núcleo auténtico de lo social son los incesantes enfrentamientos en las esferas de reconocimiento mutuo. Junto al hecho de que la integración social ocurre a través del reconocimiento que hace posible la acción comunicativa, Honneth intentará mostrar también el hecho de que tal proceso de integración social es esencialmente conflictivo, porque hay asimetrías en los procesos de interacción comunicativa en los distintos órdenes de reconocimiento.

Honneth sostiene que la decisión de Habermas de asumir una teoría de sistemas y atribuir los procesos de aprendizaje evolutivo de los sistemas 
sociales a procesos de racionalización, que toman lugar «supra-subjetivamente», lo lleva a abandonar la alternativa conceptual que encontramos en la segunda versión de su modelo teórico-comunicativo de sociedad. En vez de ir directo al concepto de «sistema social», él podría haber usado la idea de actores colectivos para evitar la idea de un sujeto unificado.

[...] los procesos de aprendizaje social mediante los cuales se desarrollan y evolucionan las sociedades no estarían entonces disponibles ni para un macro- sujeto ni para un sistema anónimo de acciones, sino para los grupos sociales que, siguiendo la estela de la elaboración comunicativa de las experiencias específicamente grupales, consiguen acceder desde el punto de vista histórico a nuevos puntos de vista y convicciones. Este planteamiento teórico habría abierto la posibilidad de interpretar el proceso de racionalización social como un desarrollo en el que los grupos sociales luchan por su modo de entender la ulterior evolución y la configuración de las instituciones sociales [...] (Honneth, 2009: 418).

Aunque pueda admitirse en Habermas la idea de esferas sociales como sistemas hipostasiados que subyace también al diagnóstico de Adorno o Foucault, es justo admitir que en Habermas, no estamos ante un modelo coercitivo del orden social en que los procesos de entendimiento moral simplemente permanecen ignorados. Justamente, lo que distingue a Habermas de la teoría crítica anterior es que ve a la acción comunicativa como el principal mecanismo de reproducción de todas las sociedades. Esto es lo que le permite criticar a Adorno o Foucault como perspectivas unidimensionales, que ven la ilustración solo como racionalización social instrumental. Pero Honneth considera que Habermas está tan atado a las convicciones básicas de la tesis tecnocrática que el dominio de la reproducción material de la sociedad lo ve como libre de normas, como una esfera de acción organizada sólo técnicamente, al punto que lo excluye de su propia teoría de la comunicación (Honneth, 2009).

Esta decisión lo hace tomar la teoría de sistemas para analizar cómo evolucionó la esfera de la economía y la política, mostrándolos como universos cerrados, enfrentados a la praxis comunicativa cotidiana. Con este dualismo, la patología social sólo puede ser concebida como la invasión del sistema desde el exterior en un mundo de la vida. Habermas así abandona en su teoría social la orientación normativa, esto es, la organización comunicativa de la reproducción material, tan importante en la tradición del marxismo crítico (Deranty, 2009). Abandona la posibilidad de una crítica justificada a formas concretas de organización de la producción económica y de la administración política. En palabras de Honneth, Habermas 
[...] pierde sobre todo -y esto, de nuevo, le convierte en un heredero de la tradición de la Teoría Crítica que aquí hemos investigado- justo ese acercamiento teórico-comunicativo que él mismo había explorado: el potencial para un entendimiento del orden social como una relación comunicativa institucionalmente mediada entre grupos integrados culturalmente que, en la medida en que el ejercicio del poder se distribuye en forma simétrica, tiene lugar en el medio de la lucha social (Honneth, 2009: 444).

La conclusión de Crítica del poder es la necesidad de retomar la ruta original de Habermas, buscando un modelo sobre el conflicto social basado en una teoría de la comunicación. Siguiendo el camino abierto y no seguido de forma consecuente por Habermas, éste debería ser concebido como un proceso que refiere a los reclamos de los individuos que pueden ser socialmente realizados. Así, esta crítica disparará la pretensión de Honneth de ofrecer una versión alternativa de una teoría comunicativa de la sociedad que pueda brindar una comprensión más correcta de la sociedad, de los conflictos cotidianos y en concreto, de sus patologías. Ella permitirá, en otros aspectos, «entender las organizaciones sociales funcionando de una forma totalitaria como frágiles construcciones que se mantienen dependientes para su existencia del consenso moral de todos sus participantes» (Honneth, 2009: 444).

\section{La respuesta de Habermas y valoración de la crítica}

Esta crítica fue retomada por Honneth en el libro que editó junto a Hans Joas en el mismo año, titulado Acción Comunicativa (Honneth y Joas, 1991). En el prefacio, Honneth reconoce que sus objeciones a Habermas allí no son originales: están inspiradas en algunos estudios críticos sobre la obra del autor entre los que menciona dos artículos publicados en aquel libro colectivo escritos por Thomas McCarthy, Hans Joas y al que podríamos sumar uno de Johannes Berger. ${ }^{3}$ Estas coincidencias teóricas muestran

3 La crítica de Honneth aparece sumamente vinculada a tres artículos: a) «Complexity and democracy: or the seducements of systems theory» de Thomas MacCarthy. Sostiene que Habermas, al adoptar el concepto funcionalista de "sistema», sucumbe a la tentación de una tradición sociológica que justamente puede ser criticada precisamente desde el enfoque inicial de Habermas. b) «The unhappy marriage of hemeneutics and funcionalism» de Hans Jonas, quien como sabemos tuvo importante influencia en su formación. Allí critica el concepto de mundo de la vida y la confianza habermasiana en el funcionalismo. Es de destacar también en dicho volumen, el artículo de Johannes Berger titulado "The linguistification of the sacred and the delinguistifation of the economy» en el que Berger cuestionaba la dicotomía entre sistema y mundo de la vida, considerando que esta rígida distinción entre acción comunicativa y estratégica y su asociación con dos dominios de acción constituía el problema central de Habermas. 
que esta crítica de Honneth se encontraba ya presente en la discusión teórica desencadenada como repercusión de la obra habermasiana. Se advertía que la expresión "colonización del mundo de la vida» podía llevar el peligro de invisibilizar la posibilidad de crisis internas en el dominio político y económico de acción, así como tampoco considerar la intrusión de orientaciones del mundo de la vida en las esferas de acción instrumental.

Un aspecto interesante para el objetivo de este artículo es que si bien Habermas nunca contestó directamente a las objeciones de Honneth, sí contamos con la réplica con que respondió a las críticas de otros autores -autores en los cuales Honneth inspiró su crítica (Habermas, 1991). Resulta sumamente valioso aquí visualizar esta réplica, entonces, como una posible respuesta indirecta a Honneth. En ella, Habermas reconoce que la distinción sistema-mundo de la vida surge de una suerte de combinación entre los modelos teóricos de Durkheim y Parsons. Y también admite que entre las razones para tal combinación figuran las dificultades metodológicas que presenta la teoría de sistemas para las ciencias sociales ${ }^{4}$ y dificultades en sus propios trabajos iniciales -en particular, Ciencia y técnica como ideología. Con esto último se refiere a un precipitado paralelismo entre sistemas de acción y tipos de acción, que Habermas reconoce a Honneth haber criticado con acierto (Habermas, 1991). Habermas admite que luego no ha trabajado suficientemente a nivel teórico la posibilidad de dicha conjunción:

En realidad, el primer intento se detuvo en seco en un confuso añadido teórico de «mundo de la vida» «sistema». Quise abordar todas estas dificultades en el segundo volumen de Teoría de la acción comunicativa. Este proyecto ha desencadenado ahora una opaca controversia que ciertamente puede ser también atribuida a no haber sido elaborada con suficiente precisión en aquel volumen (Habermas, 1991: 251).

Las lecturas que autores como Stephen Bronner han hecho de la distinción habermasiana la presentan como una distinción cuasi-ontológica entre formas de reproducción material y simbólica (Bronner, 1994), denunciando una mirada materialista de la economía y del trabajo en Habermas. No la ven como una distinción analítica, sino como una descripción de prácticas sociales opuestas y asumen que esta distinción es imposible ya que el mundo social es una interconexión de ambos.

Sin embargo, en su réplica, Habermas aclara que desde un inicio vio esta distinción como una de carácter analítico, como señalando dos aspectos de

4 Para un desarrollo mayor de la posición contraria de Habermas con respecto a una teoría pura de sistema, ver Habermas, 2005: 413-414. 
la integración de una sociedad (Habermas, 1991). Reconoce que en Ciencia y técnica como ideología cometió el error de identificar tipos de integración social con un tipo específico de acción, pero aclara que intentó corregir eso al introducir el concepto de una sociedad en dos niveles. Ni los mecanismos de integración sistémica ni los de integración social están asociados unilateralmente a un tipo de acción, y que, por ejemplo, el mundo de la vida está lejos de ofrecer una imagen de esferas de comunicación libres de poder (Habermas, 1991). En palabras de Habermas: «el mundo de la vida se desacopla solamente de los subsistemas dirigidos por los medios, pero no de los mecanismos de la integración sistémica como un todo» (Habermas, 1991: 257). Y aunque las relaciones organizacionales internas estén de algún modo neutralizadas éticamente, concede que el mundo de la vida de sus miembros, nunca completamente anulado, penetra la realidad interna de las organizaciones.

Habermas aclara que su intención fue hacer un uso sólo analítico del modelo sistémico - a la manera de Parsons-, y no uno esencialista, a la manera de Luhman; y que por ello, da una primacía metodológica al mundo de la vida -y al enfoque comunicativo. Sin embargo, explícitamente admite la crítica de McCarthy: que ambos aspectos de la sociedad, que fueron inicialmente introducidos simplemente como perspectivas distintas en la observación de los fenómenos sociales, se pierden cuando se introduce la evolución histórica como un desacople entre sistema y mundo de la vida, adquiriendo un lenguaje esencialista vinculados a dos dominios de realidad diferentes (Habermas, 1991). Habermas se reconoce culpable de un uso reificante del lenguaje en este sentido.

Hemos mencionado los argumentos de Honneth a favor de una mayor porosidad: argumenta contra la idea de subsistemas no normativos y deslingüistizados, ya que éstos siempre incluyen aspectos normativos, al menos porque siempre están íntimamente entrelazados con sistemas de dominio social. Las instituciones de la economía y del Estado nunca son exclusivamente encarnaciones de consideraciones puramente instrumentales; están enmarcados por principios práctico-políticos, los cuales dependen, a su vez de procesos comunicativos. Y lo mismo se puede decir de las instituciones económicas y administrativas (Honneth, 2009). Lo que habría hecho Habermas es «externalizar» el dominio, desde una dialéctica entre fuerzas de producción -la sociedad como sistema funcional- y la sociedad como un todo integrado normativamente que es capaz de aprendizaje moral (Deranty, 2009). Honneth buscó así un modelo alternativo que permita superar verdaderamente una teoría de sistemas para atender a las relacio- 
nes asimétricas de poder, el conflicto entre grupos por la interpretación de las normas de integración social y el rol del conflicto en la reproducción social.

También otros autores como Cohen y Arato y Claus Offe han cuestionado esta dicotomía habermasiana. Offe sostiene que el sistema económico depende en su reproducción laboral diaria de la existencia de relaciones asociativas normativamente cargadas en el proceso productivo, que integran y protegen a sus miembros y constituyen una suerte de eticidad al interior del proceso productivo (Offe, 1992). La sociología organizacional contemporánea ha aportado mucho al respecto de nuevas formas de entender el gerenciamiento de los lugares de trabajo, superando la visión extremadamente instrumental del trabajo que Habermas heredó de Weber. El proceso de trabajo capitalista está informado por reglas normativas que constituyen ambientes de acción que habilitan elevar cuestiones sobre la justicia y la obligación mutua (Offe, 1992). Habermas subestimaría el poder que tienen las instituciones sociales cargadas normativamente de poner límites éticos sobre la operativa del sistema. Por ejemplo, la legislación de ocho horas, el salario mínimo o las regulaciones sobre salud y seguridad laboral.

En el mismo sentido, Honneth desprenderá de su crítica a Habermas una concepción más rica del trabajo que defendió desde sus estudios iniciales y está desarrollando hasta hoy. Una derivación de aquella fuerte distinción analítica es que Habermas evita la noción marxista del trabajo y las connotaciones normativas que le dió Marx inspirado en Hegel, porque el trabajo se reduce a acción instrumental para la supervivencia material de la sociedad (Honneth, 1995a). Los criterios bajo los que puede juzgarse entonces son eficiencia y productividad. Aunque Honneth sostiene que la transformación posfordista de la organización del trabajo y la producción requiere de una reelaboración de la categoría marxista del trabajo (Deranty, 2009), el autor retoma a Marx en un fuerte y constante interés por los aspectos normativos del trabajo y por lo que éste significa para los sujetos, para poder hacer una verdadera concepción crítica del trabajo. Sus textos recientes de crítica del capitalismo contemporáneo van en este sentido, ya que, en opinión de Honneth, la explotación laboral es una desviación de la promesa normativa latente en el mercado (Honneth y Hartmann, 2006; Honneth, 2008).

Comparto la opinión de Cannon en cuanto a que, aunque Habermas aclara que se trata de una distinción analítica, es culpable de no considerar la compleja interconexión entre ellas (Cannon, 2001). En tal sentido, el 
concepto de patología social, cuando es explicado sólo como una invasión proveniente desde las esferas sistémicas hacia las esferas de interacción cotidiana, quita del horizonte la posibilidad de que los mundos de la vida puedan «contracolonizar» tales agresiones, y el poder comunicativo queda muy acotado. 5 También Deranty ha señalado el acierto de Honneth de esta crítica a una caída de Habermas en un lenguaje excesivamente funcionalista, y cómo la herencia de la tesis tecnocrática vuelve irrelevante el problema del poder y su distribución en la sociedad (Deranty, 2009).

A mi juicio, resulta muy útil este aporte de Honneth postulando una mayor porosidad entre estos dos ámbitos, lo que llevaría a comprender que las organizaciones, como el mercado o la administración burocrática, dependen para su funcionamiento también del consenso moral de sus participantes, a la vez que los procesos de integración social albergan una lucha o conflicto entre actores sociales por el reconocimiento de su identidad.

En consonancia con la objeción honnethiana pueden ubicarse las más recientes críticas que los exponentes españoles de la ética del discurso hacen a Habermas (García-Marzá, 2004, 2008, 2015; Cortina, 1998, 2010, 2011). El sistema de las sociedades capitalistas no se reproduce sin la inclusión permanente de elementos del mundo de la vida, ya que las acciones de gerenciamiento y administración producidas en las organizaciones dependen de decisiones y prácticas de entendimiento social. Ni el gerenciamiento ni la administración tienen posibilidad de realizarse sin acuerdo normativo de sus miembros, como suponen las teorías sistémicas de sociología de las organizaciones. Al suavizar la tajante distinción habermasiana, el mundo de la vida intervendrá en los subsistemas de la economía y el Estado. También Jesús Conill, con la idea de una «economía ética» quiere reintroducir la perspectiva de la normatividad al interior del análisis de los procesos económicos, retomando así su conjunción original (Conill, 2006). La esfera económica debe legitimarse comunicativamente y la economía debe ser capaz de cooperar en la construcción de una buena sociedad.

Y aquí es donde se introduce el último punto que nos interesa considerar. Esta dicotomía ficticia también limita, de algún modo, la concepción sobre las posibilidades de la sociedad civil en la concepción habermasiana.

5 La interconexión de ambos ámbitos está presente desde el origen mismo de ellos en la forma que Habermas lo explica. Los dos procesos que constituyen la modernización de la sociedad se presuponen el uno al otro. El mundo de la vida no puede ser modernizado sin facilitar estratégicamente la coordinación de la acción comunicativa mediante el desarrollo de los dos subsistemas. Éstos, a su vez, requieren una base institucional en el mundo de la vida que sigue estando estructurado simbólicamente, coordinado lingüísticamente y no obstante, en cierta medida, por lo menos modernizado. 
Ésta, en su perspectiva, se compone de las asociaciones y organizaciones voluntarias, no estatales y no económicas, que surgen de forma más o menos espontánea, y que arraigan las estructuras comunicativas de la opinión pública en el mundo de la vida, ya que recogen las resonancias de los problemas en los ámbitos de la vida privada y, elevándoles la voz, los transmiten al espacio de la opinión pública política. Son ciudadanos organizados que, desde la vida privada, buscan interpretaciones públicas para sus intereses y que influyen en la formación institucionalizada de la opinión y la voluntad políticas (Habermas, 2005). Habermas está con esta definición, excluyendo de la sociedad civil, tanto el poder político como el poder económico. En el ámbito de las sociedades democrático-deliberativas, la sociedad civil, protegida por el Estado de derecho, se organiza para influenciar y cercar el poder político, en la busca de soluciones para sus problemas. El poder administrativo del Estado queda en cierta forma cercado por el poder comunicativo. El espacio público-político es una caja de resonancia para problemas que han de ser elaborados por el sistema político (Habermas, 2005).

Ahora bien, en la forma que es conceptualizada por Habermas, el espacio de acción de la sociedad civil es muy limitado con respecto al sistema político institucionalizado -limitándose a ser vocera de demandas e influyente sobre la agenda del poder administrativo- y nulo con respecto a la economía, ya que ésta se considera como desarrollándose enteramente bajo la lógica instrumental.

Por ello, es que el concepto de sociedad civil ha estado al centro del debate sobre política democrática, y en particular, la formulación habermasiana ha recibido críticas como las mencionadas de Cortina y García-Marzá. La crítica fundamental que se realiza al interior de la ética del discurso, es que la concepción de Habermas es demasiado estrecha, ya que reduce la sociedad civil a las relaciones de solidaridad inmediatas, y deja fuera todo lo que suponga estrategia y competencia -o sea, el ámbito económico, mercados y empresas, y el ámbito político administrativo, de los partidos, del sistema judicial y, en general, de todo el complejo parlamentario (García-Marzá, 2004). También Adela Cortina ha señalado que la dicotomía habermasiana establecida entre, por un lado, los subsistemas político y económico, orientados al poder y al dinero respectivamente y, por otro, el mundo de la vida, asimilable a la sociedad civil y orientado a la solidaridad, es inadecuada (Cortina, 1998). De hecho, señala que el propio Habermas reconoce que el poder político sólo puede legitimarse comunicativamente por el respaldo de los ciudadanos. 
En consonancia con la tesis de Honneth de una mayor interconexión entre sistema y mundo de la vida, Cortina y García-Marzá consideran que la dimensión económica también pertenece a la sociedad civil y así reivindican, junto a una ética política, la posibilidad de una ética de la empresa, porque también en el cálculo estratégico, en los pactos, compromisos y negociaciones entre sus grupos de intereses, nos preguntamos sobre la justicia y moralidad de las actuaciones, más allá de las obligaciones jurídicas. Las decisiones y acciones empresariales también reclaman una pretensión de validez sin la cual perderían su credibilidad y confiabilidad. Para García Marzá, en la sociedad civil hay mecanismos de coordinación de la acción que están más allá de la fuerza de motivación empírica de la coacción jurídica, el dinero o el poder. Estos mecanismos incluyen tanto la estrategia y la búsqueda del interés propio, como la cooperación y la confianza. De ahí que ambos teóricos proponen ampliar el concepto de sociedad civil cuyo contenido estaría

constituido por las organizaciones e instituciones del mundo económico, por las asociaciones voluntarias en el sentido antes expuesto -comunidades adscriptivas y voluntarias, asociaciones cívicas- y por la esfera de la opinión pública. En su seno se dan la mano la racionalidad estratégica y la comunicativa, la competencia de intereses y la cooperación, el individualismo egoísta y la solidaridad. Pero todo ello dentro de un contexto de voluntariedad y pluralismo, que es su gran baza (Cortina, 1998: 379).

Como conclusión, aun no siendo la crítica de Honneth a Habermas aquí presentada una crítica "original», considero que es sumamente pertinente y potente. Sin dudas, una ventaja fundamental que presenta esta crítica frente a otras desarrolladas por el autor hacia el enfoque habermasiano, es que contamos con una suerte de posible réplica del propio Habermas. Él mismo admite no haber considerado suficientemente la interrelación entre estas dos dimensiones. La mayor porosidad que Honneth postula existe entre la esfera del sistema y del mundo de la vida, permite comprender de mejor manera los procesos sociales, y en particular, da pistas para repensar el carácter de la sociedad civil. Llama la atención, sin embargo, que Honneth, sólo se ha limitado a plantear la ambigüedad que posee el concepto y una reconstrucción de los sentidos históricos que ha tenido (Honneth, 1996), pero no se ha dedicado aún a formular una nueva conceptualización sobre la sociedad civil alternativa a la habermasiana, a la luz de tan potente crítica. 


\section{BIBLIOGRAFÍA}

Bronner, E. S. (1994): Critical theory and its theorists, Oxford, Blackwell. CANNon, B. (2001): Rethinking the normative content of critical theory. Marx, Habermas and beyond, New York, Palgrave.

Conill, J. (2006): Horizontes de economía ética, Madrid, Tecnos.

Cortina, A. (1998): «Sociedad civil» en Cortina, A. (coord.) (1998): Diez palabras clave en Filosofía Política, Navarra, Verbo Divino.

- (2010): Justicia cordial, Madrid, Trotta.

- (2011): «Ciudadanía democrática: ética, política y religión», Isegoría, 44, pp. 13-55.

Deranty, J.Ph. (2009): Beyond communication. A critical study of Axel Honneth's social philosophy, Leiden-Boston, Brill.

GaRCíA-MARZÁ, D. (1998): «Sociedad civil: una concepción radical», Recerca, 8, pp. 27-46.

- (2004): Ética empresarial. Del diálogo a la confianza, Madrid,Trotta.

- (2015): «El valor democrático de la sociedad civil», Thémata, 52, pp.93109.

McCARthy,Th.(1985): The Critical Theory of Jürgen Habermas, Cambridge, The мIT Press.

HaberMas, J. (1985): «Remarks on the concept of communicative action» en Seebass, G. y Tuomela, R. (eds.) Social Action, Dordrecht y Boston, Reidel.

- (1987): Teoría de la acción comunicativa I, Madrid,Taurus.

- (1990): Conocimiento e interés, Buenos Aires, Taurus.

- (1991): «A reply» en JoAs, H. y Honneth, A. (ed.) (1991): Communicative Action, Cambridge Massachusetts, мIт Press.

- (2003): Teoría de la acción comunicativa II, Madrid,Taurus.

- (2005): Facticidad y validez, Madrid,Trotta.

Honneth, A. y otros (1992): Cultural-Political Interventions in the Unfinished Project of Enlightenment, Cambridge, мIт Press.

Honneth, A. (1995): The fragmented world of the social, Albany, State University of New York Press.

- (1995a) «Work and instrumental action: on the normative basis of critical theory», en The fragmented world of the social. Essays in Social and Political Philosophy, Albany, State University of New York Press, pp. 44-49.

- (1995b): «Moral consciousness and class domination», en The fragmented world of the social, Albany, State University of New York Press, pp. 205-219. 
- (1996): «Concepciones de la sociedad civil», Archipiélago, 24, pp. 47 55.

- (1997): La lucha por el reconocimiento. Por una gramática de los conflictos sociales, Barcelona, Crítica.

- (2005): «Reconocimiento y justicia. Entrevista con Axel Honneth» (por Francisco Cortés Rodas), Estudios Políticos, 27, pp. 9-26.

- (2006): «Redistribución como reconocimiento» en Fraser, N. y HonNeTH, A. (2006): ¿Redistribución o reconocimiento?, Madrid, Morata.

Honneth,A. y Hartmann, M. (2006): «Paradoxes of capitalism», Constellations, 13,1, pp. 41-58.

Honneth, A. (2008): «Trabalho y reconocimento. Tentativa de uma redefiniçào", Civitas, 8, 1, pp. 46-67.

- (2009): Crítica del poder. Fases en la reflexión de una Teoría Crítica de la sociedad, Madrid, Machado Libros.

Moore, B. (1978): Injustice: the Social Bases of Obedience and Revolt, London, MacMillan.

OfFe, C. (1992): «Bindings, Shackles, Brakes: On Self-Limitation Strategies» en Honneth, A. y otros (eds.) (1992): Cultural-Political Interventions in the Unfinished Project of the Enligbtement, Cambridge, мІт Press, pp. 6394.

Honneth, A. y otros (eds.) (1992): Cultural-Political Interventions in the Unfinished Project of the Enlightement, Cambridge, мIт Press. 